\title{
Status and Prospect of Neutrino $C P$ Violation and Baryon Number Violation
}

\author{
Francesca Di Lodovico* \\ King's College London \\ E-mail: francesca.di_lodovicolkcl.ac.uk
}

\begin{abstract}
This paper addresses the status of major open questions in physics: $C P$ violation in the leptonic sector and baryon decays. $C P$ violation has not been measured in the leptonic sector yet and current long baseline neutrino experiment as $\mathrm{T} 2 \mathrm{~K}$ and $\mathrm{NO} v \mathrm{~A}$ are pursuing it whilst future experiments as DUNE and Hyper-Kamiokande will be able to give a definitive answer. An overview of the current status and future sensitivites is presented. Neutrino experiments can also be used to search for baryon decays, predicted by Grand Unification Theories, for which we present an overview of the current limits and future sensitivities.
\end{abstract}

The 4th KMI International Symposium (KMI2019)

18-20, February 2019

Nagoya, Japan

${ }^{*}$ Speaker. 
In spite of the remarkable progress in understanding neutrino properties in recent years, key open questions remain to be answered. In particular whether $C P$ symmetry is violated in the leptonic sector, as it is for the quarks. It is one of the most interesting open questions in particle physics as one of the most popular and successful explanation of the origin of the baryon asymmetry of the Universe is through the leptogenesys mechanism, that requires $C P$ symmetry violation.

Grand unification of strong, weak and electro-magnetic interactions predict a non null value of the proton decay. Since no universally accepted model has of yet emerged, a worlwide spread search is currently ongoing for proton and neutron decays.

In the following sections, we will first review the current status of the $C P$ violation studies and future perspectives and finally we will address the current limits on baryon decays as well as the future sensitivities.

\section{1. $C P$ Violation Experimental Results and Perspectives}

Long-baseline neutrino oscillation experiments are sensitive to $C P$ violation by looking at subleading terms in the appearance oscillation probability $v_{\mu} \rightarrow v_{\mathrm{e}}$. Including the $C P$ terms and low mass scale, the equation for neutrino oscillation in vacuum [1] is:

$$
\begin{aligned}
P\left(v_{\mu} \rightarrow v_{\mathrm{e}}\right) & =P 1+P 2+P 3+P 4 \\
P 1 & =\sin ^{2}\left(\theta_{23}\right) \sin ^{2}\left(2 \theta_{13}\right) \sin ^{2}\left(\Delta m_{32}^{2} L / 4 E\right) \\
P 2 & =\cos ^{2}\left(\theta_{23}\right) \sin ^{2}\left(2 \theta_{13}\right) \sin ^{2}\left(\Delta m_{12}^{2} L / 4 E\right) \\
P 3 & = \pm J \sin \left(\delta_{C P}\right) \sin \left(\Delta m_{32}^{2} L / 4 E\right) \\
P 4 & =J \cos \left(\delta_{C P}\right) \cos \left(\Delta m_{32}^{2} L / 4 E\right)
\end{aligned}
$$

where

$$
\begin{aligned}
J= & \cos \left(\theta_{13}\right) \sin \left(2 \theta_{12}\right) \sin \left(2 \theta_{13}\right) \sin \left(2 \theta_{23}\right) \times \\
& \sin ^{2}\left(\Delta m_{32}^{2} L / 4 E\right) \sin ^{2}\left(\Delta m_{12}^{2} L / 4 E\right)
\end{aligned}
$$

and the sign in $P 3$ is negative for neutrinos and positive for anti-neutrinos, respectively. For most new long-baseline accelerator experiments, the term $P 2$ is negligible, but the other three terms can all be large. Furthermore, matter effects can be relevant depending on the baseline and mass order.

Our knowledge of $\theta_{12}$ and $\Delta m_{12}^{2}$ comes from the solar and KamLAND reactor neutrino experiments whilst our knowledge of $\theta_{32}$ and $\Delta m_{32}^{2}$ comes from atmospheric, reactor and long-baseline accelerator neutrino experiments [1]. Results on $\theta_{13}$ come from reactor antineutrino disappearance and long-baseline accelerator $v_{\mathrm{e}}$ appearance experiments [1]. The interpretation of both kinds of results depends on $\Delta m_{32}^{2}$, and the accelerator results also depend on the mass order, $\theta_{13}$ and the $C P$ violating phase $\delta_{C P}$.

Accelerator and atmospheric experiments have some sensitivity to the $C P$ violation phase $\delta_{C P}$ through $P\left(v_{\mu} \rightarrow v_{\mathrm{e}}\right)$. As its term $P 3$ depends on the sign of $\Delta m_{32}^{2}$, the sensitivity to $C P$ depends on the mass order. For non-maximal $\theta_{32}$ mixing, it also depends on the octant of $\theta_{32}$, i.e. whether $\theta_{32}>\pi / 4$ or $\theta_{32}<\pi / 4$. 

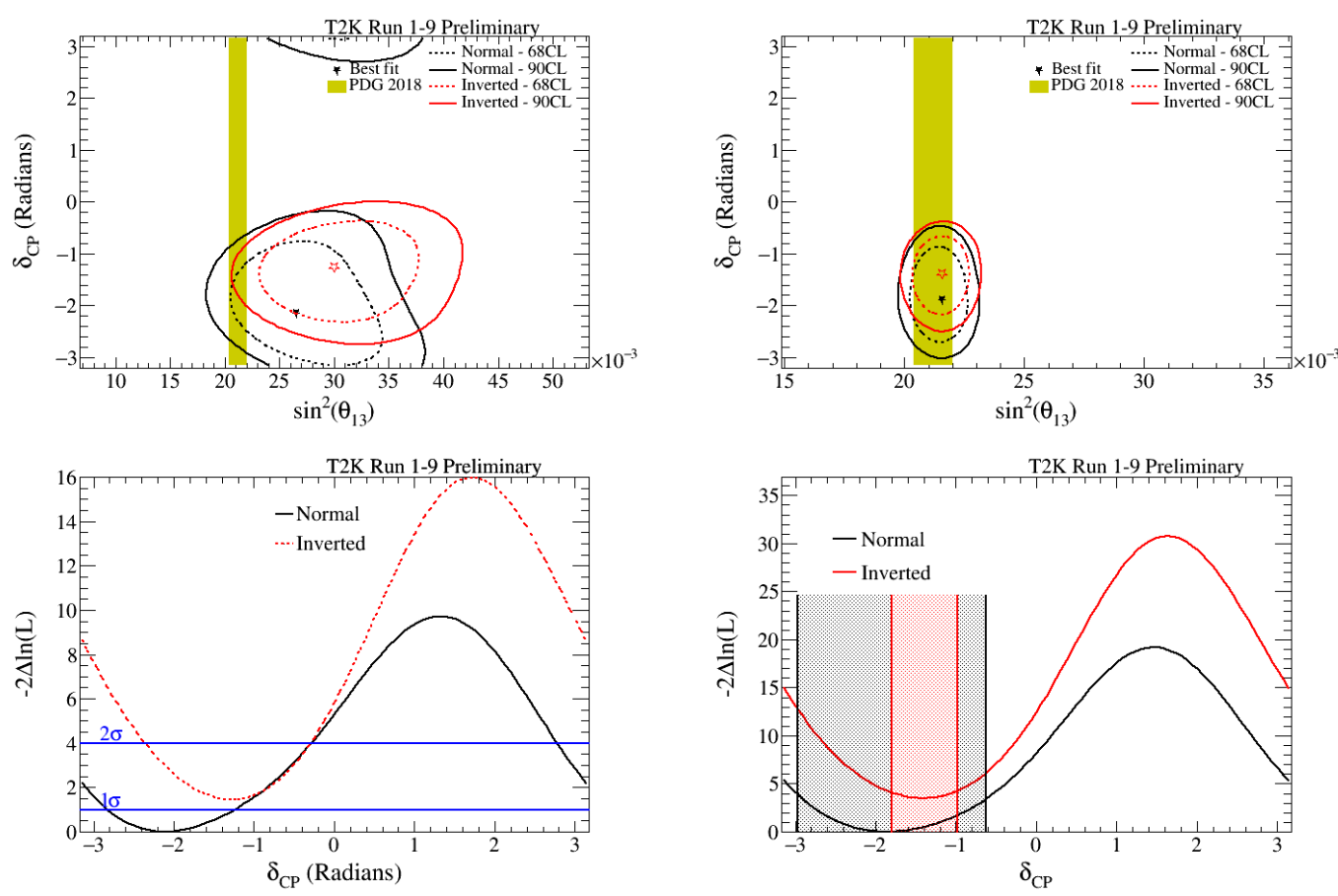

Figure 1: T2K preliminary results are shown. The first row shows the $\delta_{C P}$ versus $\sin ^{2}\left(\theta_{13}\right)$, whilst the second row the $2 \sigma$ confidence intervals for the measured $\delta_{C P} \chi^{2}$ distributions. The left plots show the results without the reactor constrain on $\theta_{13}$, whilst the right plots include the reactor constraint.

The latest results from the long baseline neutrino experiments T2K [3] and NOvA [2] are first presented and then perspectives for the future with DUNE [4] and Hyper-Kamiokande [5] discussed.

T2K presented the results of the 3 -flavour $v / v$ joint analysis on its full dataset. The 3-flavour fit is sensitive to the $C P$ violation parameter, as well as the mixing parameters $\sin ^{2}\left(2 \theta_{13}\right), \sin ^{2}\left(2 \theta_{23}\right)$, and the mass difference squared $\cos \left(\theta_{23}\right)$. T2K has little sensitivity to the mass hierarchy.

The predicted events at the far detector, Super-Kamiokande, are 272.399 \pm 13.9 (syst) for neutrino $\mu$-like, $139.5155 \pm 6.3$ (syst) for antineutrino $\mu$-like, $74.424 \pm 6.6$ (syst) for neutrino $e$ like, $17.132 \pm 1.2$ (syst) for antineutrino $e$-like and $7.026 \pm 1.3$ (syst) for neutrino $v_{\mathrm{e}} \mathrm{CC} 1 \pi$ events in Super-Kamiokande and observes respectively 243 for neutrino $\mu$-like, 140 for antineutrino $\mu$-like, 75 for neutrino $e$-like, 15 for antineutrino $e$-like and 15 for neutrino $v_{\mathrm{e}} \mathrm{CC} 1 \pi$.

The $v / \bar{v}$ analysis was performed in a framework of 3-flavour oscillations including matter effect in constant-density matter. The observed reconstructed energy spectrum of single $\mu$-like ring events and reconstructed energy versus lepton angle of single $e$-like ring events in addition to $e$-like events with an additional decay electron were used to compute the confidence intervals. All systematic parameters, for a total of 117 , as well as the oscillation parameters which were not parameters of interest considered in the analysis were marginalised.

From the fit of the Run 1-9 dataset corresponding to an integrated J-PARC neutrino beam exposure of $1.4938 \times 10^{21}$ protons-on-target $(\mathrm{POT})$ in neutrino mode and $1.6346 \times 10^{21}$ POT in antineutrino mode the favoured value of $\delta_{C P}$ is -1.885 , close to $-\pi / 2$. The Feldman-Cousins 
method is used to construct confidence intervals with neither over nor under coverage from which the significance to exclude the hypothesis of $C P$ conservation is greater than 2 standard deviations. The results from the fit are shown in Fig. 1.

Concerning NOvA, the other running long baseline neutrino experiment, the results are based a combined analysis of neutrino $\left(8.85 \times 10^{20} \mathrm{POT}\right)$ and antineutrino $\left(6.91 \times 10^{20} \mathrm{POT}\right)$ data full data sample. Neutrino oscillations parametrization, fits, predictions and interpretation of the results were done within the standard oscillation model of 3 active neutrino flavours of electron, muon and tau neutrino. To obtain oscillation parameters, a simultaneous fit of joint $v_{\mathrm{e}}+v_{\mu}$ and both neutrino and antineutrino data was performed. Systematic uncertainties are incorporated as nuisance parameters with Gaussian penalty term, appropriately correlated between all the data sets.

A total of $102 \bar{v}_{\mu}$ candidate events at the far detector, reflecting a significant suppression from the unoscillated expectation of 476 , were observed. A total of $27 \bar{v}_{\mu}$ $\rightarrow \bar{v}_{\mathrm{e}}$ candidate events with an estimated background of $10.3_{-0.5}^{+0.6}$ were observed corresponding to a $4.4 \sigma$ excess over the predicted background. This observation is the first evidence of $\bar{v}_{\mathrm{e}}$ appearance in a $\bar{v}_{\mu}$ beam over a long baseline. These new antineutrino data

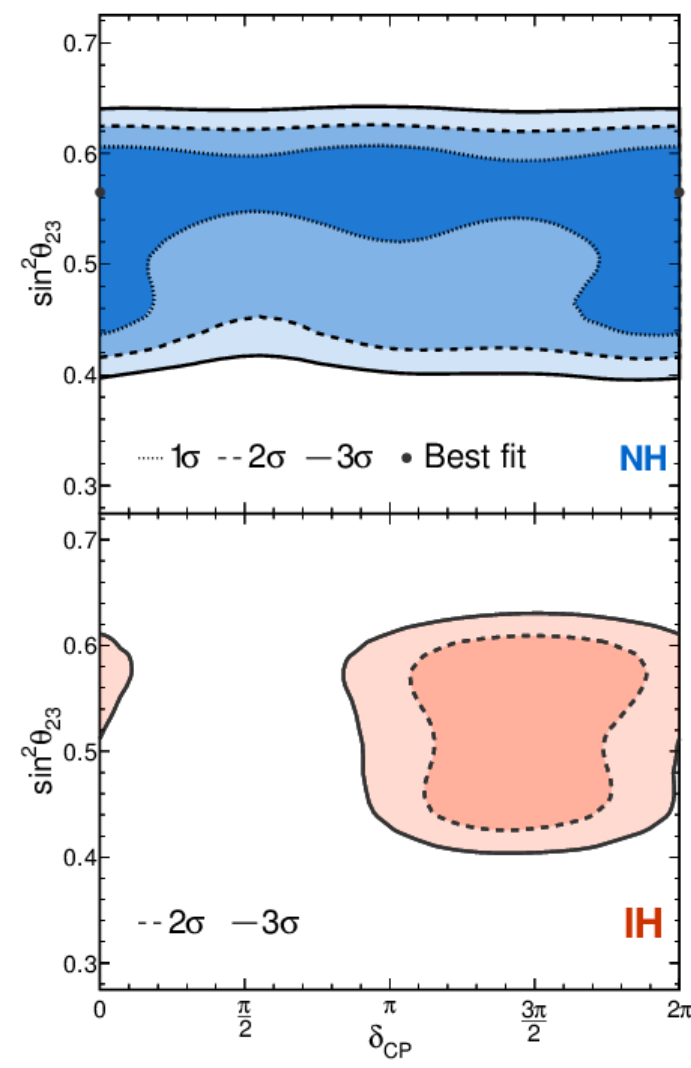

Figure 2: The $a \sigma, 2 \sigma$, and $3 \sigma$ contours in $\sin ^{2}\left(\theta_{23}\right)$ versus $\delta_{C P}$ in the normal hierarchy ( $\mathrm{NH}$, top panel) and inverted hierarchy (IH, bottom panel). The bestfit point is shown by a black marker. are analysed together with $113 v_{\mu}$ and $58 v_{\mu} \rightarrow v_{\mathrm{e}}$ candidates from the previous data set. The best-fit point is found for the normal hierarchy with $\theta_{32}$ in the upper octant where $2 \ln L=157.1$ for 175 degrees of freedom (goodness-of-fit $p=0.91$ from simulated experiments). The measured values of $\theta_{32}$ and $\Delta m_{32}^{2}$ are consistent with the previous NOvA measurement that used only neutrino data, and are consistent with maximal mixing within $1.2 \sigma$. Confidence intervals for the oscillation parameters are determined using the unified approach.

Fig. 2 shows the allowed regions in $\sin ^{2}\left(\theta_{23}\right)$ and $\delta_{C P}$. These results exclude most values near $\delta_{C P}=\pi / 2$ in the inverted mass hierarchy by more than $3 \sigma$; specifically the intervals between 0.04 to $0.97 \pi$ in the lower $\theta_{32}$ octant and 0.04 to $0.91 \pi$ in the upper octant. The data prefer the normal hierarchy with a significance of $1.9 \sigma(p=0.057, \mathrm{CLs}=0.091)$ and the upper $\theta_{32}$ octant with a significance of $1.6 \sigma(p=0.11)$, profiling over all other parameter choices.

Both T2K and NOvA will run for the next years up to around 2025-2026 with increasing beam power, a new refurbished near detector for $\mathrm{T} 2 \mathrm{~K}$ and new reduced systematics errors. NOvA will benefit from the results of a testbeam. In both cases improved analyses techniques will be used. 
Furthermore, T2K and NOvA have started to discuss to provide future combined sensitivities.

Beyond $\sim 2025$ the future long baseline experiments, the Deep Underground Neutrino Experiment (DUNE), and Hyper-Kamiokande will start to take data with the aim to provide the final measurement of the $C P$ violation parameter.

The DUNE experiment will measure muon-neutrino disappearance and electron and tau neutrino appearance, with both neutrino and anti-neutrino beams, providing information on several neutrino oscillation parameters in a single experiment. At the chosen baseline distance of $1300 \mathrm{~km}$, the effects of matter-induced asymmetry and the $C P$ violation phase $\delta_{C P}$ impact the neutrino spectra differently, thus the two effects can be disentangled. In 10 years of operation, DUNE will reach $5 \sigma$ sensitivity to the neutrino mass ordering for any value of $\delta_{C P}$ and it will be able to discover $C P$ violation in the leptonic sector at $3 \sigma$ for $50 \%$ of $\delta_{C P}$ values. The sensitivities are shown in Fig. 3. Improvements are expected with recent analyses on full simulation, using automated energy reconstruction and deep-learning based event selection, such as Convolutional Neural Networks (CNN).
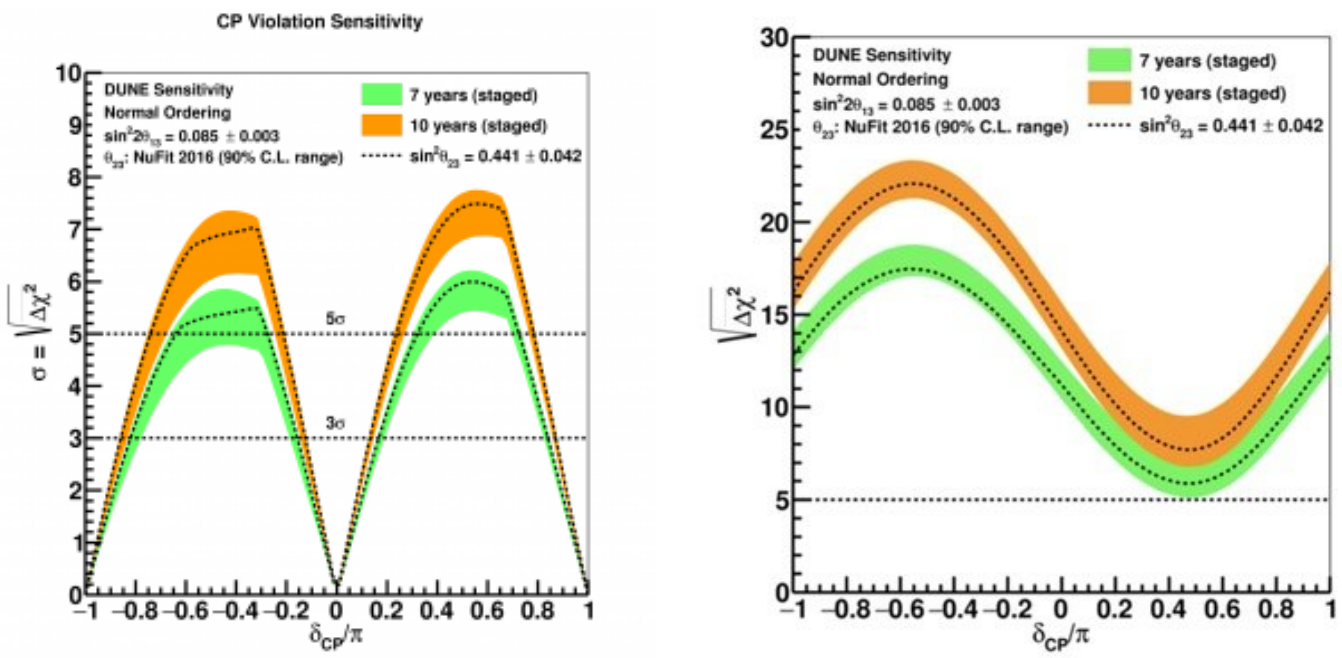

Figure 3: Sensitivity of DUNE to the neutrino mass ordering (left) and the leptonic $C P$ violation phase (right). The green and orange bands correspond to an exposure of 300 and $556 \mathrm{kton} \times \mathrm{MW} \times \mathrm{y}$, respectively.

Combining an intense and high quality neutrino beam from J-PARC, the huge mass and high performance of the Hyper-Kamiokande detector, a highly capable near/intermediate detector complex, and the full expertise obtained from the ongoing T2K/Super-Kamiokande experiments, Hyper-Kamiokande will probe the $C P$ violation in the lepton sector and new physics with neutrino oscillations.

The Hyper-Kamiokande sensitivity is estimated based on a framework developed in the T2K experiment. The analysis is the same, except for the update of the systematic uncertainty estimate. A binned likelihood analysis based on the reconstructed neutrino energy distribution is performed using both $v_{\mathrm{e}}\left(\bar{v}_{\mathrm{e}}\right)$ appearance and $v_{\mu}\left(\bar{v}_{\mu}\right)$ disappearance samples simultaneously. The systematic uncertainty is estimated based on the experience of $\mathrm{T} 2 \mathrm{~K}$, with an extrapolation considering improvement expected in the Hyper-Kamiokande era. Correlations of systematics between energy bins and flavours are taken into account using an error matrix. Figure 4(left) shows the expected significance to exclude $\sin \delta_{C P}=0$ (the $C P$ conserved case). $C P$ violation in the lepton sector can 
be observed with more than $3(5) \sigma$ significance for $78(62) \%$ of the possible values of $\delta_{C P}$. Figure 4(right) shows the $68 \% \mathrm{CL}$ uncertainty of $\delta_{C P}$ as a function of the running time. The value of $\delta_{C P}$ can be determined with an uncertainty of $7.2^{\circ}$ for $\delta_{C P}=0^{\circ}$ or $180^{\circ}$, and $21^{\circ}$ for $\delta_{C P}= \pm 90^{\circ}$.
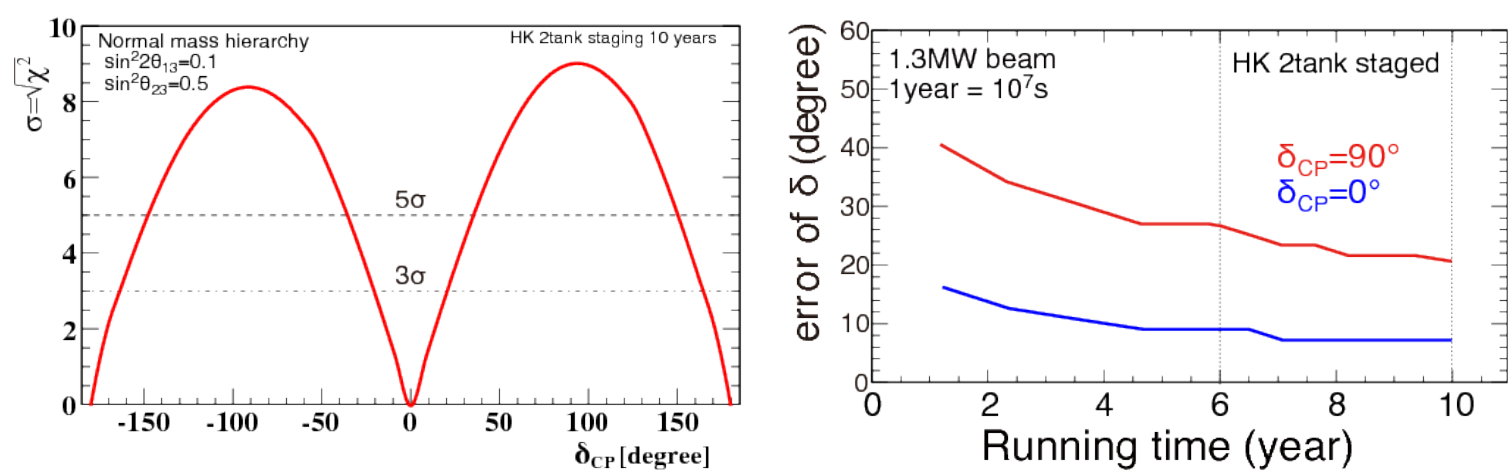

Figure 4: (Left) Expected significance to exclude $\sin \delta_{C P}=0$ for normal mass hierarchy. (Right) Expected $68 \% \mathrm{CL}$ uncertainty of $\delta_{C P}$ as a function of running time.

Finally, there is a proposal, ESSnuSB [6], to use the neutron spallation source, ESS, in Sweden to also provide a neutrino beam. The current work is predominantly performed on the realisation of a neutrino beam from the spallation source. This experiment is being investigated and a timeline of the decade after DUNE and Hyper-Kamiokande is assumed.

\section{Baryon Decay Experimental Searches and Perspectives}

One of the three Sakharov conditions [7] to explain the baryon asymmetry of the universe is the baryon number violation, which is believed to have played an important role during the formation of the universe. Proton decay and the decay of bound neutrons are observable consequences of the violation of baryon number.

The Standard Model (SM) [1] has been successful in accounting for many experimental results. However, the SM offers no guidance on the unification of the strong and electroweak forces, and has several other open questions. Various attempts have been made to resolve the shortcomings by unifying the strong and electroweak interactions in a single larger gauge group, i.e. a Grand Unified Theory (GUT) [1] . GUTs are motivated by the apparent convergence of the running couplings of the strong, weak, and electromagnetic forces at a high energy scale $\left(10^{15} 10^{16} \mathrm{GeV}\right)$. Energy scales this large are out of the reach of accelerators but may be probed by virtual processes such as those that govern particle decay. A general feature of GUTs is the instability of nucleons by baryon number violating decay. Therefore nucleon decay experiments are direct experimental tests of the general idea of grand unification. In GUTs, nucleon decay can proceed via exchange of a massive gauge boson between two quarks. The favoured gauge-mediated decay mode in many GUTs is $p \rightarrow e^{+}+\pi^{0}$. GUT models incorporating supersymmetry (SUSY-GUTs) raise the GUT scale, suppressing the decay rate of $p \rightarrow e^{+}+\pi^{0}$, thereby allowing compatibility with the experimental limit. However, SUSY-GUTs introduce dimension five operators that enable the mode $p \rightarrow k^{+}+\bar{v}$ to have a high branching fraction and short partial lifetime. 
In Fig. 5, we show 90\% CL lifetime limits by Super-Kamiokande and earlier experiments compared with representative lifetime ranges predicted by various GUTs. Hyper-Kamiokande has a mass that will allow to improve on the current limits by Super-Kamiokande [9]. DUNE is also sensitive to nucleon decay but due to its smaller mass compared to Hyper-Kamiokande, it is competitive mainly in modes with distinctive final state tracks such as those involving kaons. Similarly for the JUNO experiment [8].

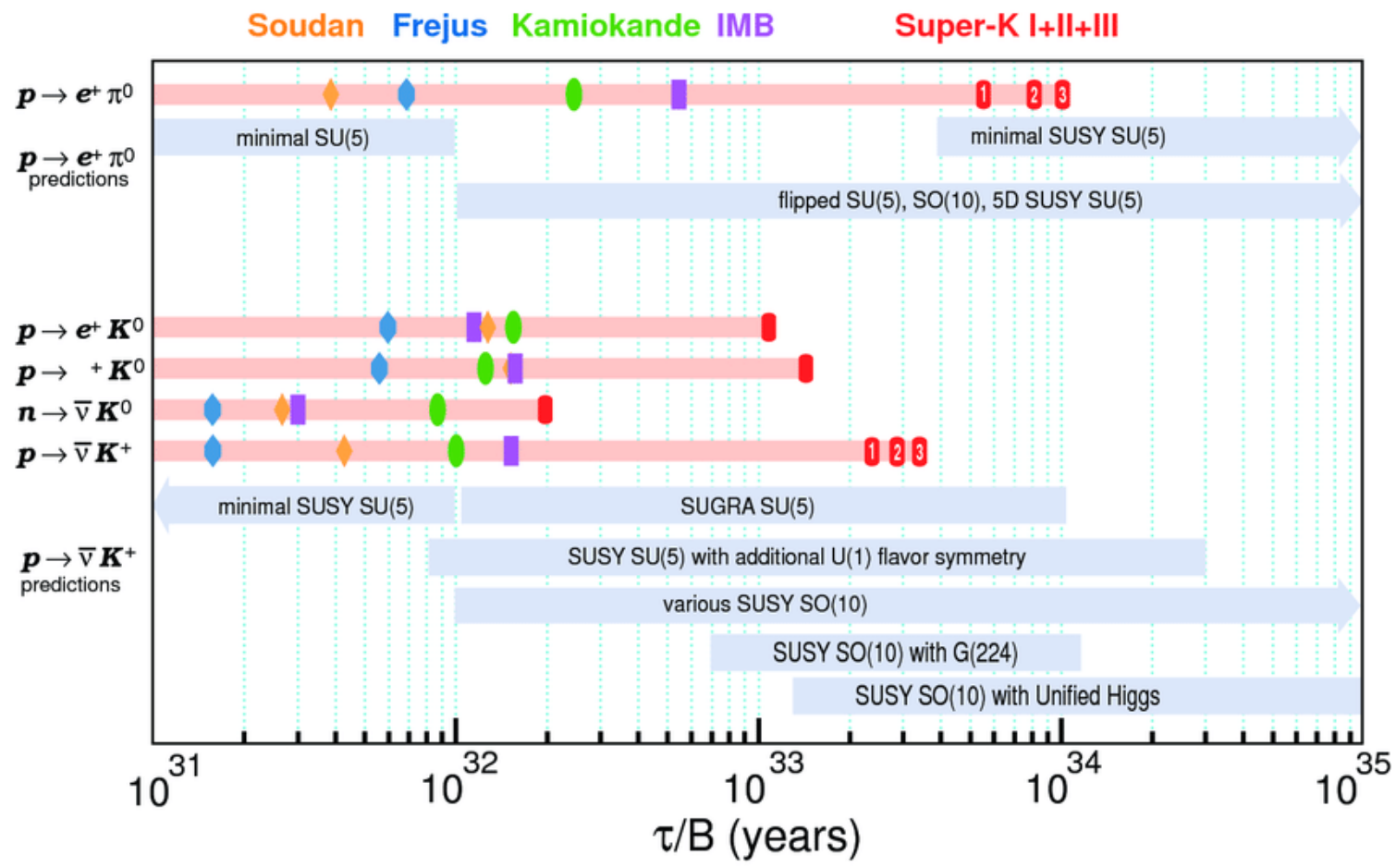

Figure 5: Proton decay lifetime limits compared to lifetime ranges predicted by Grand Unified Theories. The upper section is for $p \rightarrow e^{+}+\pi^{0}$, most commonly caused by gauge mediation. The lower section is for SUSY motivated models, which commonly predict decay modes with kaons in the final state. The marker symbols indicate published limits by experiments, as indicated by the sequence and colors on top of the figure.

Generally, nucleon decay may occur through multiple channels and ideally, experiments would reveal information about the underlying GUT by measuring branching ratios (see Fig. 6). Similarly to Super-Kamiokande, it is a strength of Super-Kamiokande that it is sensitive to a wide range of nucleon decay channels.

Finally, the expected discovery potential for Hyper-Kamiokande compared to Super-Kamiokande, DUNE and JUNO is shown in Fig 7.

\section{Conclusions}

A review of the current status and future prospects for two of the most challenging open questions in physics are presented in this paper. The status of the leptonic $C P$ violation parameter is reviewed, which could lead to understanding the origin of the baryon asymmetry in the Universe. 


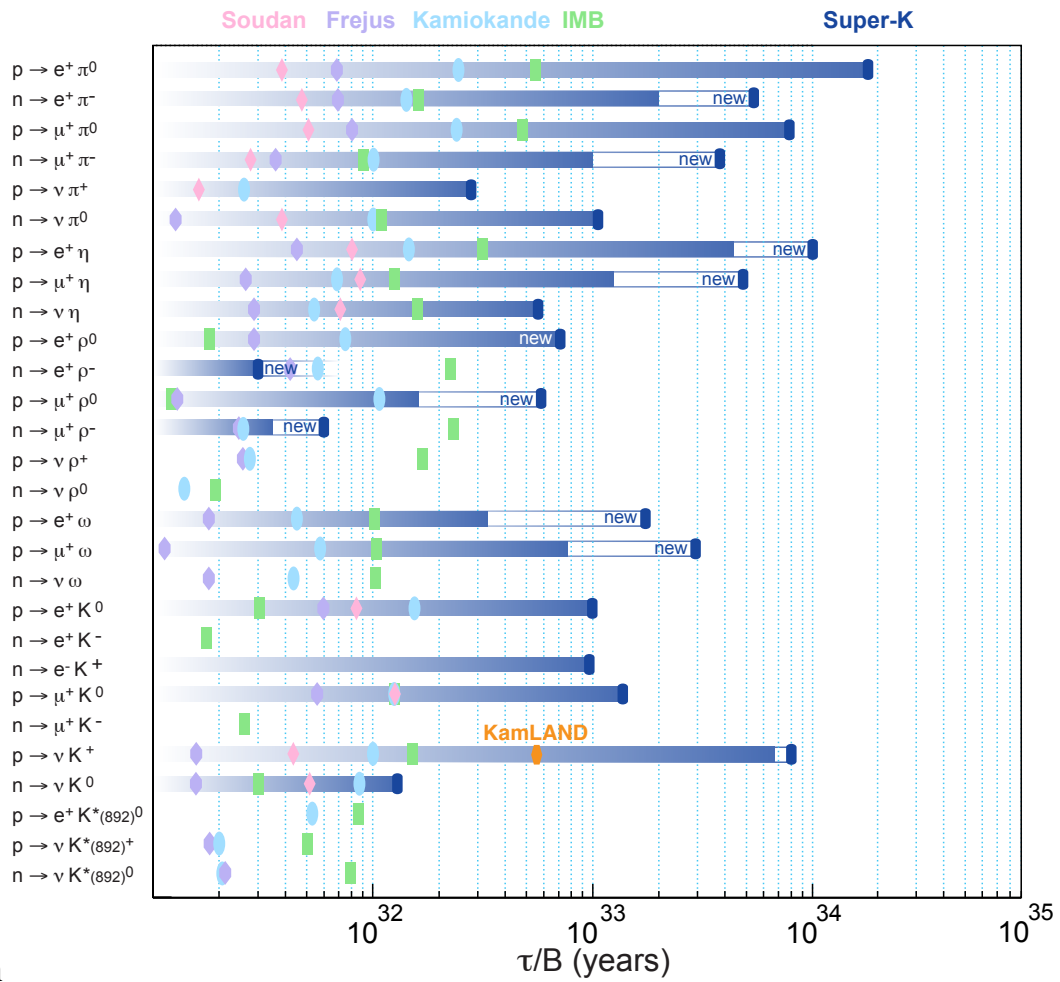

Figure 6: Summary of lifetime limits on different final states.
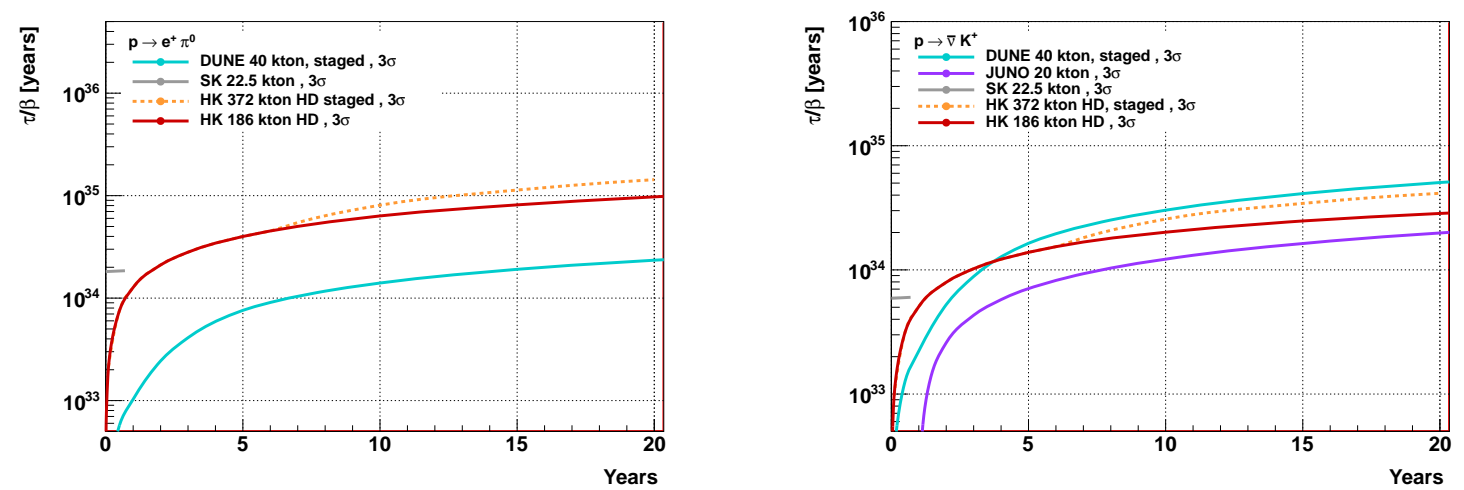

Figure 7: Comparison of the $3 \sigma$ discovery potential as a function of year or Hyper-Kamiokande for $p \rightarrow$ $e^{+}+\pi^{0}$ (left) and $p \rightarrow K^{+}+\bar{v}$ (right) as well as that of the 40 kton DUNE detector based on [10] and the 20 kton JUNO detector based on [8].

The status of the baryon decay searches is also presented, which would be an indication of GUT theories if observed. New experiments will be able to further address these issues in the next decades.

\section{Acknowledgement}

The author would like to thank the organisers for the invitation, sponsorship and very active 
conference.

\section{References}

[1] M. Tanabashi et al. [Particle Data Group], Phys. Rev. D 98 (2018) no.3, 030001. doi:10.1103/PhysRevD.98.030001

[2] M. A. Acero et al. [NOvA Collaboration], arXiv:1906.04907 [hep-ex].

[3] K. Abe et al. [T2K Collaboration], Phys. Rev. Lett. 121 (2018) no.17, 171802 doi:10.1103/PhysRevLett.121.171802 [arXiv:1807.07891 [hep-ex]] plus preliminary statistical update with full statistics.

[4] B. Abi et al. [DUNE Collaboration], arXiv:1807.10334 [physics.ins-det].

[5] K. Abe et al. [Hyper-Kamiokande Collaboration], arXiv:1805.04163 [physics.ins-det].

[6] E. Baussan et al. [ESSnuSB Collaboration], Nucl. Phys. B 885 (2014) 127 doi:10.1016/j.nuclphysb.2014.05.016 [arXiv:1309.7022 [hep-ex]].

[7] A. D. Sakharov, Pisma Zh. Eksp. Teor. Fiz. 5 (1967) 32 [JETP Lett. 5 (1967) 24] [Sov. Phys. Usp. 34 (1991) no.5, 392] [Usp. Fiz. Nauk 161 (1991) no.5, 61]. doi:10.1070/PU1991v034n05ABEH002497

[8] F. An et al. [JUNO Collaboration], J. Phys. G 43 (2016) no.3, 030401 doi:10.1088/0954-3899/43/3/030401 [arXiv:1507.05613 [physics.ins-det]].

[9] K. Abe et al. [Super-Kamiokande Collaboration], Phys. Rev. D 95 (2017) no.1, 012004 doi:10.1103/PhysRevD.95.012004 [arXiv:1610.03597 [hep-ex]].

[10] R. Acciarri et al. [DUNE Collaboration], arXiv:1512.06148 [physics.ins-det]. 